\title{
BMJ Open Factors associated with breast cancer screening participation among women in mainland China: a systematic review
}

\author{
Zijing Wu, ${ }^{\oplus}$ Yu Liu, ${ }^{1}$ Xiaohan Li, ${ }^{1}$ Bing Song, ${ }^{1}$ Cuiping Ni, ${ }^{1}$ Frances $\mathrm{Lin}{ }^{2}$
}

To cite: Wu Z, Liu Y, Li X, et al. Factors associated with breast cancer screening participation among women in mainland China: a systematic review. BMJ Open 2019;9:e028705. doi:10.1136/ bmjopen-2018-028705

- Prepublication history and additional material for this paper are available online. To view these files, please visit the journal online (http://dx.doi org/10.1136/bmjopen-2018028705).

Received 20 December 2018 Revised 06 August 2019 Accepted 09 August 2019

Check for updates

(C) Author(s) (or their employer(s)) 2019. Re-use permitted under CC BY-NC. No commercial re-use. See rights and permissions. Published by BMJ.

${ }^{1}$ School of Nursing, China Medical University, Shenyang,

China

${ }^{2}$ School of Nursing and Midwifery, Menzies Health Institute Queensland, Griffith University, Gold Coast, QLD, Australia

Correspondence to

Dr Cuiping Ni; cpni@cmu.edu.cn

\section{ABSTRACT}

Objectives Although detecting breast cancer at an early stage through screening has been clearly shown to be an effective strategy, the screening participation rate in China remains low. This systematic review sought to synthesise the current evidence to identify factors associated with breast cancer screening participation among women in mainland China.

Design This study was a systematic review.

Data sources Studies were collected from PubMed, PsycINF0, CINAHL, EMBASE and three major Chinese databases, specially China National Knowledge Infrastructure, Chongqing VIP and Wanfang Data.

Eligibility criteria All included papers were original research studies with a longitudinal or cross-sectional study design that considered associated factors of breast cancer screening participation among women in mainland China.

Data extraction and synthesis Study selection, data extraction and quality assessment were conducted independently by two reviewers, involving a third to help reach a consensus when necessary. Primary outcomes of interest included factors associated with breast cancer screening participation.

Results A total of 19 studies were included in this review. Based on these studies, geographical region, a personal history of breast disease, past screening behaviours for breast disease, physical examination and the availability of medical specialists/equipment for breast examination were consistently associated with participation in breast cancer screening, while residential area, ethnicity and attitude towards breast cancer screening appeared to be associated with participation in breast cancer screening. There were additionally some factors with even more limited evidence with which to ascertain their association with screening participation.

Conclusions There exists a wide range of factors that influence breast cancer screening participation in mainland China. These findings could help to inform future research and policy efforts.

\section{INTRODUCTION}

Breast cancer is the most commonly diagnosed cancer in women worldwide and one of the most common causes of death from cancer globally. ${ }^{1}$ In China, breast cancer is now the most common cancer as well as the leading cause of cancer-related death. ${ }^{2}$

\section{Strengths and limitations of this study}

This is the first systematic review to analyse the empirical evidence for factors associated with breast cancer screening participation among women in mainland China.

- There was a lack of consistency in the definition and measurement of related factors among the included studies, which makes the comparison of results difficult.

- The level of evidence from the included studies was low, with all of the included studies being cross-sectional studies, which suggests that more research with rigorous methodologies is required to better understand the factors associated with breast cancer screening participation among women in mainland China.

Although the breast cancer incidence rate in China is lower than those in Western countries, breast cancer incidence in China has still increased dramatically since the 1990s. ${ }^{3}$ In 2012, approximately 187213 new cases of breast cancer were diagnosed, with 47984 deaths attributed to the disease in China that same year. ${ }^{4}$ Furthermore, in China, the mean age of women diagnosed with breast cancer is $45-55$ years of age, which is younger than those who typically receive the diagnosis in Western countries. ${ }^{2}$

Detecting breast cancer at an early and treatable stage through screening has been shown to reduce mortality and improve survival. ${ }^{56}$ The breast cancer screening guidelines issued by the US Preventive Service Task Force (2016) suggest women aged 50-74 years undergo biennial screening mammography (MMG) and also recommend screening be completed for women aged 40-49 years if the benefits of screening outweigh the risks. ${ }^{7}$ Female breast cancer mortality rates have been declining over the past two decades in the USA in part due to improvements in early detection by screening and treatment. ${ }^{1}$ Currently, in China, there is no nationwide breast cancer screening programme, and 
the guidelines for breast cancer screening practices are different from those in Western countries. In addition to clinical breast examination (CBE), ultrasonography has been employed as an alternative option to MMG. The Chinese Anti-Cancer Association published guidelines for breast cancer screening in 2017, which suggest that women aged 40 years or older attend CBE in combination with either MMG or ultrasonography annually. ${ }^{8}$ However, despite the available clinical evidence regarding the importance of screening, the participation rate of breast cancer screening remains low in China. A large-scale national study conducted in China reported the overall participation rate of breast cancer screening among women aged $35-69$ years was only $22.5 \% .^{9}$

While a number of systematic reviews reporting factors related to breast cancer screening have been published in the last decade, most of them only included studies conducted in developed countries. ${ }^{10-15}$ To our knowledge, there is no systematic review published to date on the Chinese population concerning factors contributing to breast cancer screening. Previous reviews suggested that factors influencing breast cancer screening participation included sociodemographic factors (eg, age, education, marital status, ethnicity and income), personal attitudes and beliefs, knowledge, acculturation, access to health, social support, social influence and culture norms. ${ }^{10-15}$ Over the past five decades, although China has undergone significant development and remarkable change in its socioeconomic hierarchy, a disparity in healthcare services still exists between the rural and urban areas of China because of the large and widely dispersed population. Also, in Chinese culture, women hold different beliefs about the body, illness and health as compared with those in Western culture. ${ }^{16}$ Therefore, a review on factors contributing to breast cancer screening participation among Chinese women is needed. Furthermore, considering the differences in the healthcare systems of Taiwan, Hong Kong and mainland China, this review will only include studies conducted involving mainland Chinese women. The purpose of this systematic review was, therefore, to synthesise current evidence and identify factors associated with breast cancer screening participation among women in mainland China. This review will hopefully inform future policy efforts and research on interventions to improve breast cancer screening participation among women in mainland China.

\section{METHODS}

\section{Search strategy}

The review was performed according to the Preferred Reporting Items for Systematic Reviews and Meta-Analyses guidelines. ${ }^{17}$ The research question was what are the factors associated with breast cancer screening participation among women in mainland China? We searched the following databases: PubMed, PsycINFO, CINAHL, EMBASE and three major Chinese databases, specially China National Knowledge Infrastructure, Chongqing
VIP and Wanfang Data. Search terms used included a combination of the following: 'breast cancer', 'screening', 'detection', 'mammogra*', 'clinical breast exam*', 'China', 'Chinese', 'factor', 'predict*', 'associat*', 'relat*' and 'determin*' (see online supplementary file 1, table 1). All database searches were performed from the time of database inception to 30 July 2019. Additional articles were identified by examining the reference lists of retrieved studies.

\section{Selection criteria}

Studies were included if they (1) defined the method of breast cancer screening as MMG, ultrasound or CBE; (2) addressed associated factors of breast cancer screening participation; (3) employed a longitudinal or cross-sectional design; (4) included women who were aged 18 years or older; (5) compared groups of women who participated and did not in breast cancer screening; (6) were conducted in mainland China and (7) were published in Chinese or English.

Studies were excluded if they met the following criteria: (1) were not original studies, such as commentaries, editorials, conference abstracts, opinion statements, practice guidelines or case reports; (2) were studies on screening tests performed after the diagnosis of breast cancer; (3) were studies on genetic testing and counselling in breast cancer; (4) were studies focused on general cancer screening but not specifically on breast cancer screening and (5) were studies that included breast self-examination (BSE), where the results for breast cancer screening were not clearly separated from those of BSE. With regard to BSE, other research suggests it neither improves early diagnosis rate nor reduces mortality. ${ }^{18}$ Nevertheless, as it could somewhat improve public awareness, BSE is considered an important part of health education. ${ }^{8}$

The study selection process involved two steps. An initial screening of titles and abstracts was conducted against the inclusion/exclusion criteria. Then, full texts of the articles identified during the initial screening were examined. Two authors ( $\mathrm{ZW}$ and $\mathrm{CN}$ ) independently completed these two steps. A third reviewer (YL) was available to resolve disagreements between the two reviewers related to the two steps of the screening process where necessary.

\section{Quality assessment}

Quality assessments of the included articles were conducted by two independent reviewers (ZW and CN) using the criteria developed by Johannesen and LoGiudice, ${ }^{19}$ who provided a simple appraisal tool to measure the main factors affecting study quality. The quality assessment tool consisted of eight items that must be met, including (1) clearly defined inclusion and exclusion criteria; (2) minimised selection bias; (3) a good response rate (ie, $\geq 80 \%$ ); (4) well-defined outcome; (5) outcome measured with a valid and reliable instrument; (6) well-defined risk factors; (7) risk factors measured with valid and reliable instruments and (8) findings 
adjusted for confounding risk factors (see online supplementary file 1, table 2). For each item, if the criterion was achieved, one point was awarded, with the highest score possible being eight points. Included studies were further classified into the two groups of high quality ( $\geq 5$ points) or low quality ( $\leq 4$ points). ${ }^{19}$ Any disagreements were resolved through discussion with the third reviewer (YL).

\section{Data extraction}

Data extraction was independently performed by one author (ZW) using a data extraction form and checked by a second author $(\mathrm{CN})$. The information extracted included first author, year of publication, study design, the place where the study was performed, sampling method, the place where the women were recruited, sample size, age, breast cancer screening method, screening participation rate, conceptual framework, identified factors associated with breast cancer screening participation, and the estimates of associations between factors and screening participation (table 1 and online supplementary file 1 , table 3).

\section{Data synthesis}

A meta-analysis was not performed due to observed heterogeneity in terms of the measurement of related factors, the screening method and the reference period between the screening visit and the questionnaire assessment across the studies. Instead, a narrative synthesis was performed.

\section{Patient and public involvement}

No patients or members of the public were involved in this review.

\section{RESULTS}

\section{Search outcomes}

A total of 2538 articles were initially identified and retrieved from the databases mentioned above. After removing duplicates and further screening the titles and abstracts, 42 papers were included in the full-text screening step. A total of 19 papers were subsequently included in this review. Figure 1 shows the process of study selection.

\section{Study characteristics}

Table 1 summarises the 19 included studies. All studies employed a cross-sectional design to examine factors associated with breast cancer screening participation. Across the studies, there were differences in the breast cancer screening method used and the length of the reference period between the screening visit and the questionnaire assessment as well as the measurement of related factors. Eight studies defined the method of breast cancer screening as CBE, MMG or ultrasound ${ }^{90-26}$; five studies defined it as MMG or ultrasound ${ }^{27-31}$; and one study defined it as MMG only. ${ }^{32}$ Five studies examined the associated factors of $\mathrm{CBE}$ and $\mathrm{MMG}^{33-37}$ Most studies did not state how many years passed between the screening visit and the questionnaire assessment. The participation rate of breast cancer screening was measured by self-reported data in all studies. The sample size of the included studies varied from 326 to 72511 individuals. Most of the studies reported data from only one city, while two national studies included samples from most geographical regions in China, ${ }^{924}$ and one study included participants from multiple geographical areas in China. ${ }^{23}$ The health belief model was used as a theoretical framework in five studies, ${ }^{22} 31333637$ while one study was guided by the Knowledge-Attitude-Practice Model, ${ }^{35}$ and another study used Andersen's behavioural model of health service use. ${ }^{26}$ The remaining 12 studies did not report using any specific theoretical framework.

Regarding the quality assessment, all studies achieved a quality score of 5 or more points out of a maximum of 8 points and were rated as being of a high quality. The most common observed problem was lower validity and reliability of the outcomes measure, with all 19 studies using self-reported data. The second common problem noted was sampling bias, with nearly half of the studies $(\mathrm{n}=10)$ using convenience sampling, followed by a lack of a clear description regarding whether related factors were measured with valid and reliable instruments $(n=5)$. Additionally, one study did not clearly define factors associated with breast cancer screening, ${ }^{25}$ and one study had a low response rate of $77.62 \%{ }^{26}$

\section{Factors associated with breast cancer screening participation}

Table 2 presents the factors associated with breast cancer screening participation identified in the included studies. These associated factors are organised into five categories, including sociodemographic factors, health-related factors, knowledge, perceptions and cues to action.

\section{Sociodemographic factors}

There were nine sociodemographic factors identified in the included studies. Among them, three factors displayed a clear relationship with breast cancer screening participation (table 3). First, geographical region displayed a consistent association with participation in breast cancer screening: women from Eastern China had a higher participation rate of breast cancer screening when compared with those from Western China. ${ }^{9324}$ In two nationwide studies with random samples, living in Eastern China increased the participation rate, with an OR of $1.5,{ }^{24}$ while living in Western China decreased the participation rate, with an OR of $0.54 .^{9}$ Residential area and ethnicity also demonstrated a pattern indicating association with participation in breast cancer screening. Women living in rural areas were less likely to attend breast cancer screening as compared with those living in urban areas. ${ }^{9} 202133$ Ethnic minority women were also less likely to undergo breast cancer screening in comparison with Han women (the largest ethnicity in China). ${ }^{923} 32$ The evidence for relationships involving age, education level, employment, marriage, household income or medical insurance was 


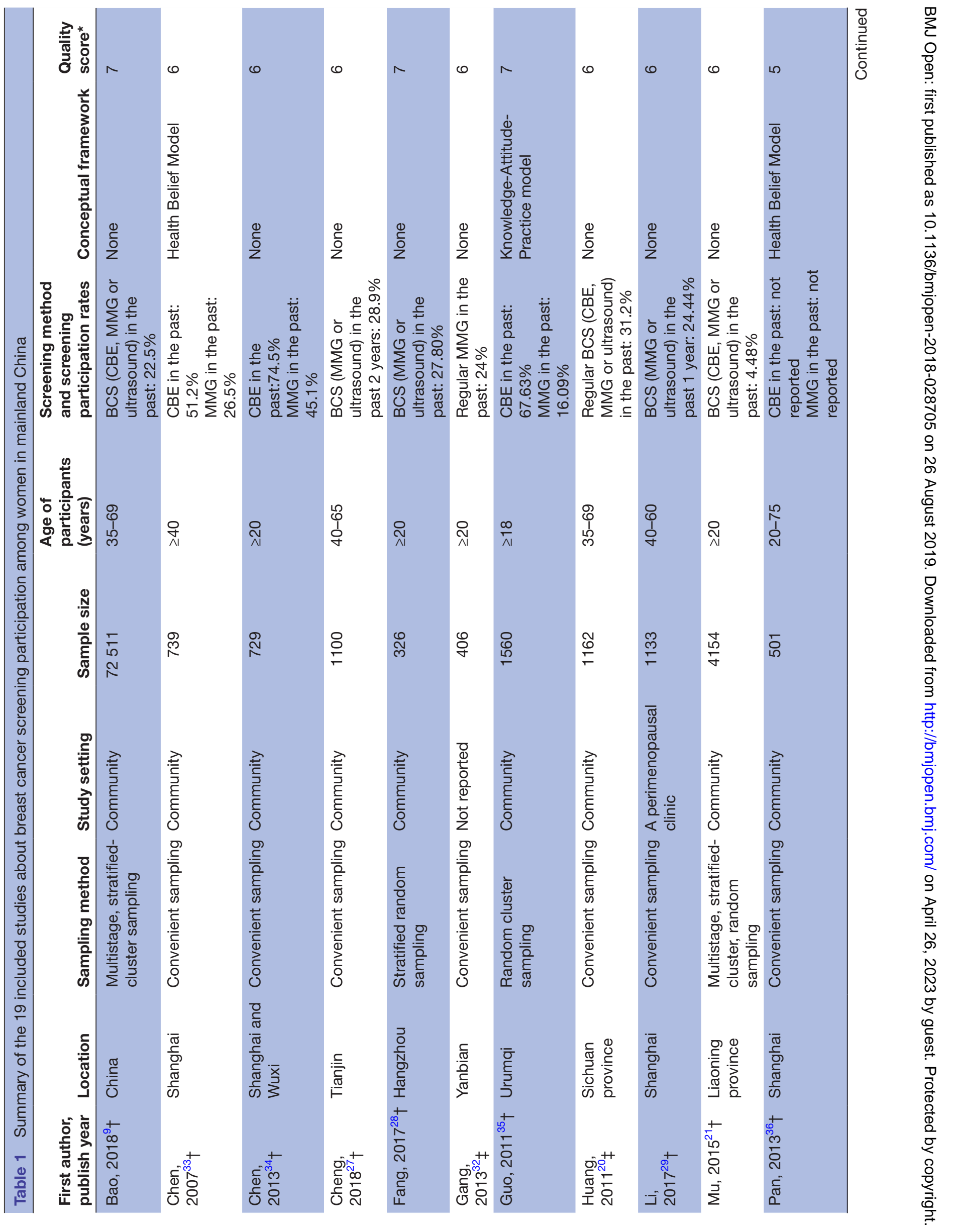




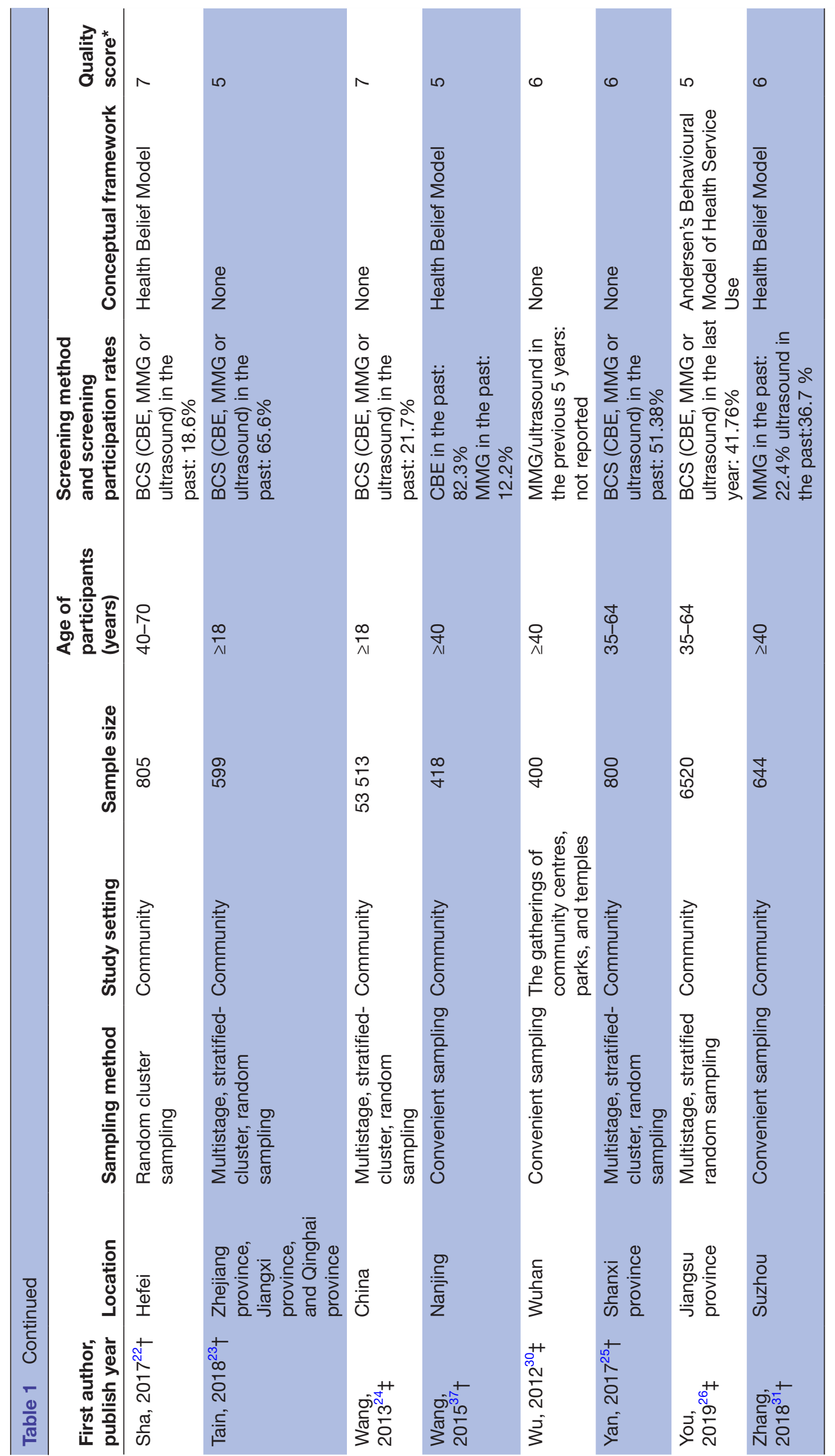

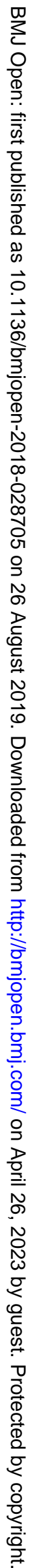




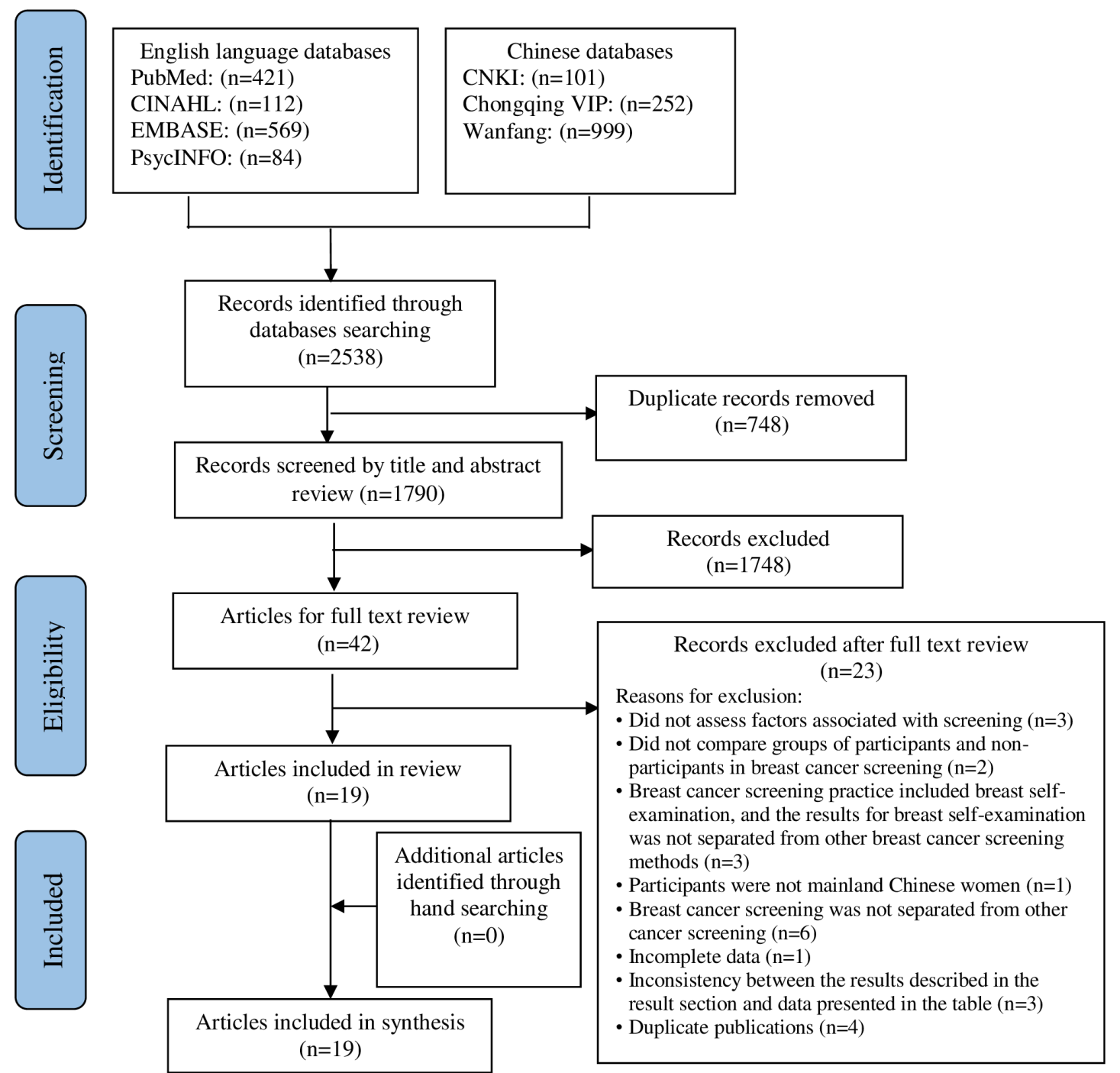

Figure 1 Flow chart of the study selection process. CNKI, China National Knowledge Infrastructure.

more unclear, because different studies reported inconsistent findings.

\section{Health-related factors}

There were eight health-related factors identified in the included studies. Having a personal history of breast disease was consistently associated with participation in breast cancer screening (table 4).$^{20273-35}$ For example, in a study involving 1560 community women from Urumqi, having a personal history of breast disease increased the rate of undergoing CBE (OR 13.05) and MMG (OR 4.20). ${ }^{35}$ In contrast, there was no consistent association pattern reported for family history of breast cancer, early menarche, perceived health status, family history of other cancer, history of induced abortion, body mass index or history of childbirth.

\section{Knowledge}

For the factor of knowledge, there was no clear association with participation in breast cancer screening. Four studies reported that women with a high level of knowledge about the risk factors, symptoms, and screening methods of breast cancer were more likely to undergo breast cancer screening in comparison with those with a lower level of knowledge. ${ }^{202728}{ }^{36}$ However, the other four studies did not observe any effect of breast cancer knowledge on participation in breast cancer screening. ${ }^{23} 303537$

\section{Perceptions}

There were eight factors related to perceptions identified from the included studies. Attitude towards breast cancer screening displayed the most consistent association with participation in breast cancer screening. Women with a positive attitude towards breast cancer screening were more likely to undergo breast cancer screening (table 4). ${ }^{23} 2837$ For example, in Tian et als study containing random population samples from three provinces, a positive attitude towards breast cancer screening increased the participation rate for both urban women (OR 2.58) and rural women (OR 7.97) alike. ${ }^{23}$ Separately, there was no consistent pattern of association reported for either perceived barriers, perceived benefits, perceived susceptibility or perceived severity of breast 
Table 2 Factors and associations (or non-associations) with participation in breast cancer screening among women in mainland China

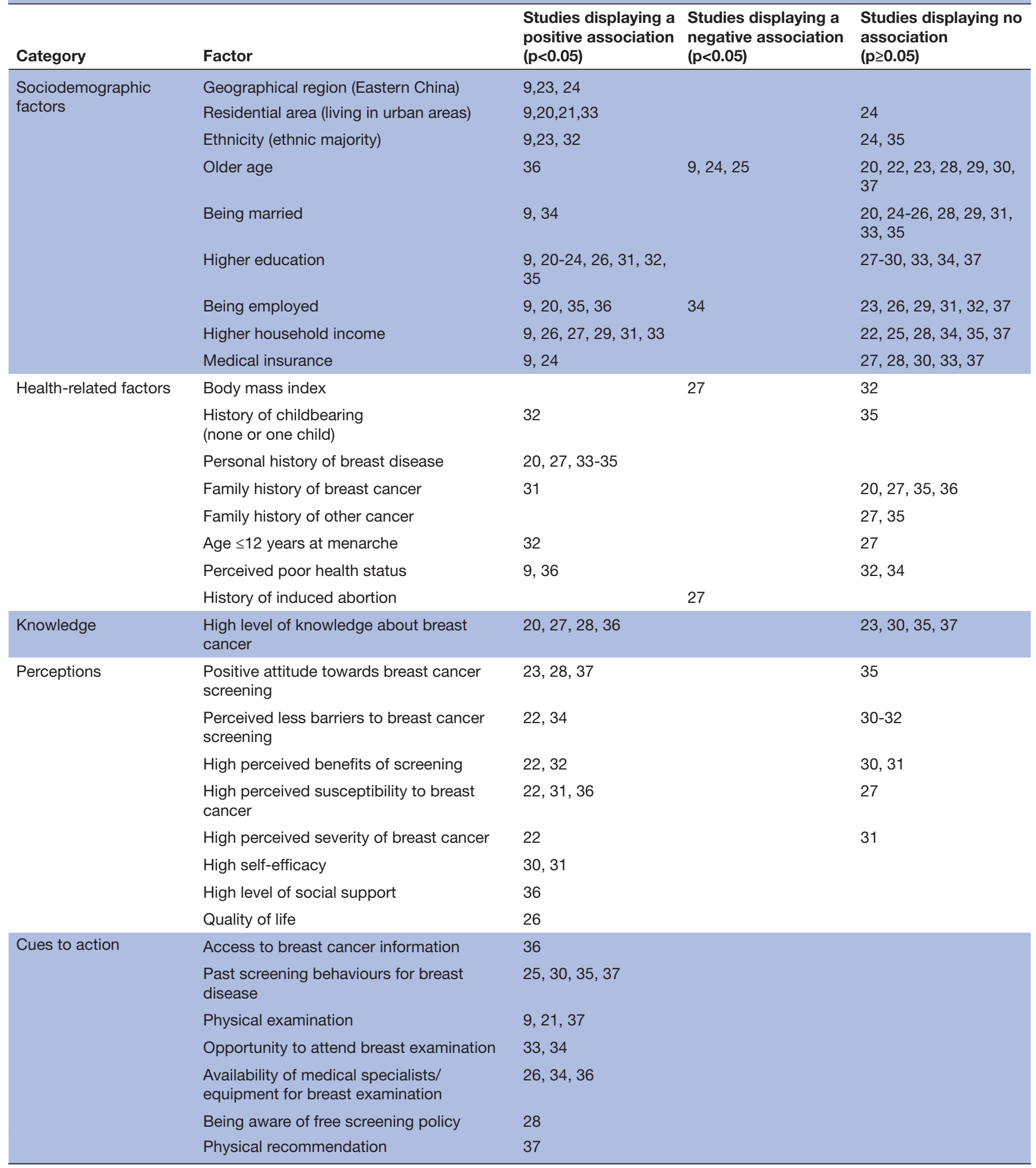

cancer. Although self-efficacy, ${ }^{30} 31$ social support ${ }^{36}$ and quality of life ${ }^{26}$ were reported to be positively associated with breast cancer screening participation, these three factors were examined only in one or two studies, which provided weak evidence.

\section{Cues to action}

A total of seven factors of cues to action were identified from the included studies. All seven factors were reported positively associated with participation in breast cancer screening, and three of these factors were examined in 


\begin{tabular}{|c|c|c|c|c|c|c|}
\hline First author, publish year & \multicolumn{2}{|c|}{ Geographical region } & \multicolumn{3}{|c|}{ Residential area } & $\begin{array}{l}\text { Ethnicity } \\
\text { OR (95\% Cl) }\end{array}$ \\
\hline Bao, $2018^{9}$ & $\begin{array}{l}\text { Central China } \\
\text { versus Eastern } \\
\text { China }\end{array}$ & $\begin{array}{l}0.51(0.28 \text { to } \\
0.90)\end{array}$ & $\begin{array}{l}\text { Rural versus } \\
\text { Urban }\end{array}$ & $\begin{array}{l}0.91 \text { ( } 0.83 \text { to } \\
0.99)\end{array}$ & $\begin{array}{l}\text { Other ethnicity } \\
\text { versus Han }\end{array}$ & $\begin{array}{l}0.88(0.79 \text { to } \\
0.98)\end{array}$ \\
\hline Chen, $2007^{33}$ & & & $\begin{array}{l}\text { Urban versus } \\
\text { Suburban }\end{array}$ & $\begin{array}{l}\text { For } \mathrm{CBE} \\
1.67(1.21 \text { to } \\
2.30)\end{array}$ & & \\
\hline Gang, $2013^{32}$ & & & & & $\begin{array}{l}\text { Being Chinese } \\
\text { versus Korean- } \\
\text { Chinese }\end{array}$ & $\begin{array}{l}2.20(1.22 \text { to } \\
3.95)\end{array}$ \\
\hline $\mathrm{Mu}, 2015^{21}$ & & & $\begin{array}{l}\text { Urban versus } \\
\text { Rural }\end{array}$ & $\begin{array}{l}4.54(2.63 \text { to } \\
7.84)\end{array}$ & & \\
\hline \multirow[t]{3}{*}{ Tian, $2018^{23}$} & & $\begin{array}{l}\text { For urban } \\
\text { women }\end{array}$ & & & & For rural women \\
\hline & $\begin{array}{l}\text { Central China } \\
\text { versus Western } \\
\text { China }\end{array}$ & $\begin{array}{l}3.32(1.70 \text { to } \\
6.48)\end{array}$ & & & $\begin{array}{l}\text { Non-Han versus } \\
\text { Han }\end{array}$ & $\begin{array}{l}0.32 \text { (0.14 to } \\
0.94)\end{array}$ \\
\hline & $\begin{array}{l}\text { Eastern China } \\
\text { versus Western } \\
\text { China }\end{array}$ & $\begin{array}{l}10.57 \text { (4.98 to } \\
22.41)\end{array}$ & & & & \\
\hline Wang, $2013^{24}$ & $\begin{array}{l}\text { Middle China } \\
\text { versus Western } \\
\text { China }\end{array}$ & $1.0(0.9$ to 1.3$)$ & & & & \\
\hline
\end{tabular}

CBE, clinical breast examination.

at least three studies, yielding much stronger evidence (table 5). For the factor of past screening behaviours for breast disease, the adoption of one breast cancer screening method was positively associated with having other breast screening methods. ${ }^{25} 3035$ For example, Wu et als study found that undergoing monthly BSE and a $\mathrm{CBE}$ in the past 2 years increased the rate of undergoing MMG/ultrasound (OR 4.53 and OR 3.04, respectively). ${ }^{30}$ For physical examination, women who received a physical examination in the past were more likely to participate in breast cancer screening. ${ }^{91}{ }^{21}$ As an example, a study including 4154 women from Liaoning province reported that having a physical examination in the past increased the breast cancer screening participation rate, with an OR $8.05 .^{21}$ Availability of medical specialists/equipment for breast examination was also positively related to breast cancer screening. ${ }^{26}{ }^{34}{ }^{36}$ Specially, in a study with 6520 women from Jiangsu Province, the availability of female medical faculty when requested in township facilities improved the participation rate, with an OR 2.98. ${ }^{26}$

\section{DISCUSSION}

To our knowledge, the present study is the first systematic review to identify factors associated with breast cancer screening participation among women in mainland China. Findings from this review could help to identify subgroups of women who are less likely to attend breast cancer screening visits and guide the development of effective health programme aiming to promote breast cancer screening practices among mainland Chinese women. 
Table 4 Main factors related to health and perceptions associated with participation in breast cancer screening among women in mainland China

\begin{tabular}{|c|c|c|c|c|}
\hline \multirow{2}{*}{$\begin{array}{l}\text { First author, } \\
\text { publish year }\end{array}$} & \multicolumn{2}{|c|}{ Personal history of breast disease } & \multicolumn{2}{|c|}{ Attitude towards breast cancer screening } \\
\hline & Group & OR $(95 \% \mathrm{Cl})$ & Group & OR $(95 \% \mathrm{Cl})$ \\
\hline \multirow[t]{2}{*}{ Chen, $2007^{33}$} & Yes versus No & $\begin{array}{l}\text { For CBE: } 4.07 \text { ( } 2.45 \text { to } \\
6.78)\end{array}$ & & \\
\hline & Yes versus No & $\begin{array}{l}\text { For MMG: } 3.91 \text { (2.58 to } \\
5.93 \text { ) }\end{array}$ & & \\
\hline Huang, $2011^{20}$ & No versus Yes & $\begin{array}{l}\text { For never attending } \\
\text { screening: } \\
3.60 \text { (1.02 to } 12.65)\end{array}$ & & \\
\hline \multirow[t]{2}{*}{ Chen, $2013^{34}$} & Yes versus No & For CBE: 5.93 & & \\
\hline & Yes versus No & For MMG: 3.78 & & \\
\hline Cheng, $2018^{27}$ & Yes versus No & $\begin{array}{l}\text { For never attending } \\
\text { screening: } 0.26\end{array}$ & & \\
\hline Fang, $2017^{28}$ & & & $\begin{array}{l}\text { Positive attitude versus Negative } \\
\text { attitude }\end{array}$ & 2.15 (1.18 to 4.69$)$ \\
\hline \multirow[t]{2}{*}{ Guo, $2011^{35}$} & Yes versus No & $\begin{array}{l}\text { For CBE: } 13.05 \text { ( } 8.57 \text { to } \\
19.85)\end{array}$ & & \\
\hline & Yes versus No & $\begin{array}{l}\text { For MMG: } 4.20 \text { (3.04 to } \\
5.79)\end{array}$ & & \\
\hline \multirow[t]{2}{*}{ Tian, $2018^{23}$} & & & $\begin{array}{l}\text { Positive attitude versus Negative } \\
\text { attitude }\end{array}$ & $\begin{array}{l}\text { For urban women: } 2.58(1.38 \\
\text { to } 4.83)\end{array}$ \\
\hline & & & $\begin{array}{l}\text { Positive attitude versus Negative } \\
\text { attitude }\end{array}$ & $\begin{array}{l}\text { For rural women: } 7.97 \text { (4.08 to } \\
15.57 \text { ) }\end{array}$ \\
\hline Wang, $2015^{37}$ & & & $\begin{array}{l}\text { High level of health belief versus } \\
\text { Low level }\end{array}$ & For MMG: 3.71 (1.43 to 9.65$)$ \\
\hline
\end{tabular}

CBE, clinical breast examination; MMG, mammography.

The included studies vary in terms of targeted age groups, sample sizes and methods of breast cancer screening. Also, the factors associated with screening attendance for different methods of breast cancer screening were measured by different tools among the various studies. All cross-sectional studies used self-reported screening practices without verification. Only seven studies were guided by a theoretical framework, and many of them focused on the sociodemographic variables influencing breast cancer screening participation, with a few factors regarding health condition, knowledge, perceptions and cues to action similarly included in the analysis. Due to the difference in the definitions and measurements of associated factors, it is difficult to accurately compare these factors across the studies. Furthermore, the findings about the factors influencing breast cancer screening participation should be interpreted with caution due to the availability of breast cancer screening programme. Although the Chinese government has introduced breast cancer screening efforts such as a programme promoting free examination for two cancers (breast and cervical cancer) among rural women and the Cancer Screening Programme in Urban China, these initiatives are accessible by only a finite number of women, leaving a substantial number of women in China still unable to access such programme. ${ }^{38}$

The associations between breast cancer screening participation and sociodemographic characteristics among women were complex. Geographical region was consistently reported to influence breast cancer screening participation in two studies including a nationally representative population sample ${ }^{924}$ and one study that included a population sample from three provinces of China. ${ }^{23}$ Women from Western China were less likely to participate in breast cancer screening when compared with those from Eastern China. Across China, there are major differences in economic and sociocultural development, and the healthcare system. The economic status and healthcare services of Western China are the least developed, which may explain the difference in screening participation rate. Living in urban areas also appeared to positively predict participation in breast cancer screening, which further confirms an economic influence on breast cancer screening participation. This finding suggests that developing effective breast cancer screening programme must consider local economic conditions and low-cost screening strategies.

A lack of medical insurance has been found to be a barrier to breast cancer screening participation in recent 
Table 5 Main factors of cues to action associated with participation in breast cancer screening among women in mainland China

\begin{tabular}{|c|c|c|c|c|}
\hline \multirow{2}{*}{$\begin{array}{l}\text { First author, } \\
\text { publish year }\end{array}$} & \multicolumn{2}{|c|}{$\begin{array}{l}\text { Past screening behaviours for breast } \\
\text { disease }\end{array}$} & \multicolumn{2}{|c|}{ Physical examination } \\
\hline & Group & OR (95\% Cl) & Group & OR $(95 \% \mathrm{Cl})$ \\
\hline \multirow[t]{4}{*}{ Bao, $2018^{9}$} & & & $\begin{array}{l}\text { Time since } \\
\text { last physical } \\
\text { examination }\end{array}$ & \\
\hline & & & $\begin{array}{l}1-3 \text { years versus } \\
<1 \text { year }\end{array}$ & $\begin{array}{l}0.90(0.83 \text { to } \\
0.97)\end{array}$ \\
\hline & & & $\begin{array}{l}>3 \text { years versus } \\
<1 \text { year }\end{array}$ & $\begin{array}{l}0.87(0.79 \text { to } \\
0.96)\end{array}$ \\
\hline & & & $\begin{array}{l}\text { Never physical } \\
\text { examination } \\
\text { versus }<1 \text { year }\end{array}$ & $\begin{array}{l}0.30(0.28 \text { to } \\
0.32)\end{array}$ \\
\hline
\end{tabular}

Chen, $2013^{34}$

Professional medical

For CBE: 2.80 staffs in the hospital: yes versus no

Professional equipment For MMG: 1.38 in the hospital: yes versus no

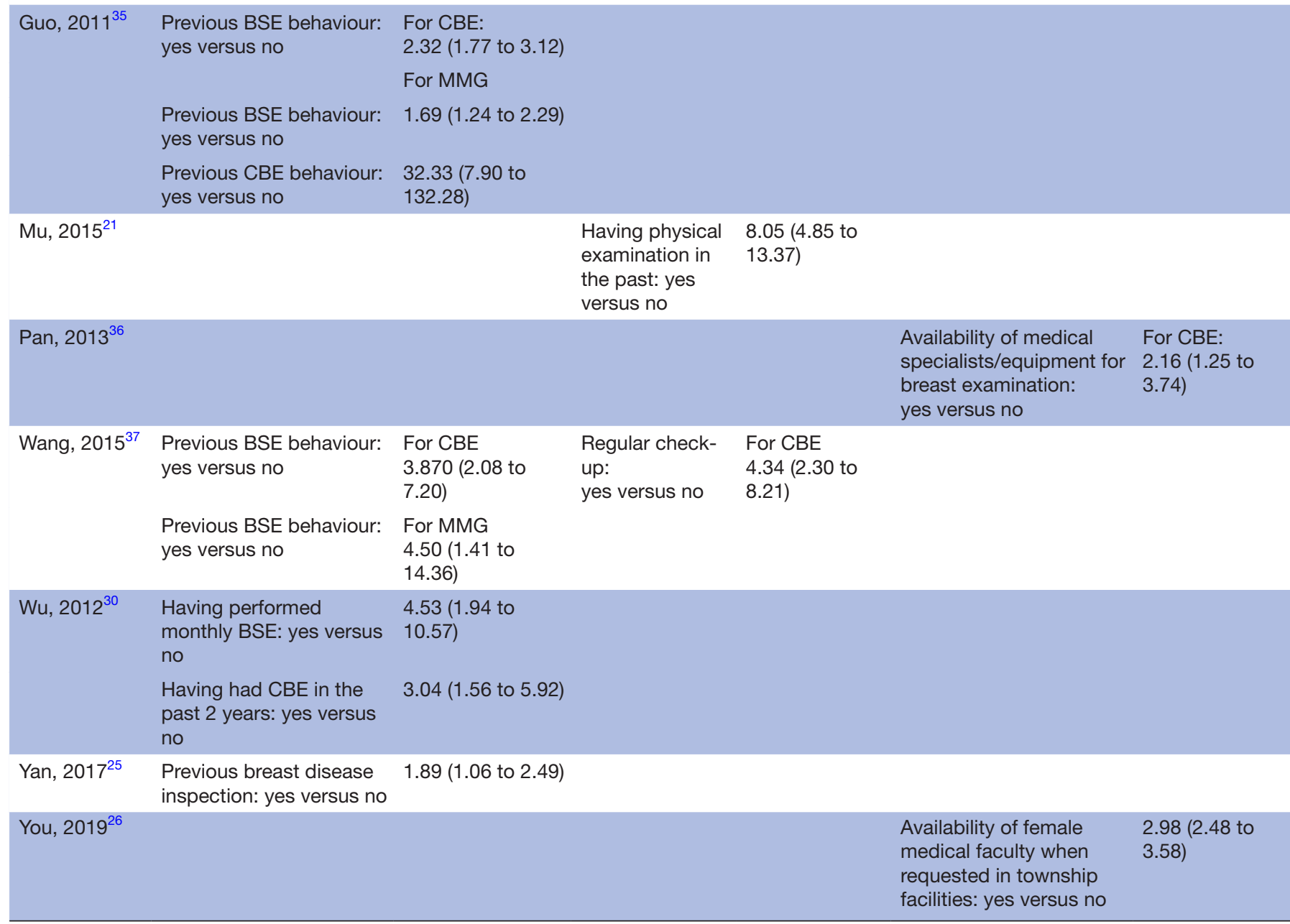

BSE, breast self-examination; CBE, clinical breast examination; MMG, mammography.

reviews ${ }^{10} 1439$; however, evidence regarding medical insurance as a predictor of breast cancer screening participation was weak in this review, due to inconsistent findings among the different studies. This could be explained by urban-rural disparities in medical insurance. ${ }^{40}$ It is likely that some of the effects of medical insurance were captured in the effects of the residential area component. However, in China, most breast cancer screening is 
opportunistic, and women have to pay for the screening test, as it is not covered by medical insurance. A lack of reimbursement for accessing the service can hinder the uptake of breast cancer screening. In the USA, the combination of health insurance and government assistance promotes widespread adoption of breast cancer screening. ${ }^{5}$ This suggests that the Chinese government should consider expanding medical insurance coverage to include breast cancer screening, and further studies are needed to explore the economic feasibility. Besides, special attention should be paid to women of ethnic minorities, because our data show that the participation rates among these women were much lower than those living in mainstream society. To improve the participation rates of screening among ethnic minorities, it is likely necessary to tailor interventions with culturally accepted languages and health promotion channels according to ethnic characteristics.

A personal history of breast disease, past screening behaviours for breast disease, physical examination and the availability of medical specialists/equipment for breast examination were consistently reported to influence breast cancer screening participation. These findings are in accordance with the findings from a review on MMG screening among American women ${ }^{14}$ and a review on breast cancer screening among Korean-Americans. ${ }^{10}$ Women with these characteristics have more opportunities to visit health providers and get a recommendation about taking the test for diagnosis. The encounter can act as an opportunity to educate women about the importance of breast cancer screening. It means that health providers need to play a more active role in promoting breast cancer screening during their contact with women.

Although recent reviews report knowledge about symptoms, risk factors and screening methods of breast cancer is an important predictor of participation in breast cancer screening, ${ }^{12} 13153941$ this review did not provide strong evidence regarding the effect of knowledge due to inconsistent findings between different studies. This might be explained by poor awareness and knowledge about breast cancer among Chinese women, which was reported by several studies. ${ }^{42-44}$ Given the rapid increase in breast cancer incidence, improving the knowledge about breast cancer screening as one of the educational components should be a high priority in the national health agenda. Nowadays, in China, the primary information sources of breast cancer screening are mass media, relatives or friends and healthcare providers. ${ }^{35}$ However, a systematic review of cancer screening interventions among Asian women reported that print materials and media campaigns alone are ineffective. They are likely to work if combined with interventions targeting health providers. ${ }^{45}$ Thus, it is vital to collaborate with health providers to explore more effective ways to convey information on breast cancer screening to women, especially those who have not previously participated in any screening practice.

Associations between perceptional factors and breast cancer screening participation are consistent with the health belief model, which suggests that self-efficacy, perceived benefits, perceived susceptibility, perceived severity and perceived barriers can affect breast cancer screening participation. However, these items' predictive powers were evaluated in only a few studies. In line with the previous review among Asian women, ${ }^{1546}$ the attitude about breast cancer is a significant predictor. Limited knowledge about breast cancer screening can contribute to a negative attitude, which further reflects the importance of education. However, due to the cultural characteristics of modesty and privacy, Chinese women may feel embarrassed to discuss breast issues or show their breasts to others, especially a male physician. ${ }^{16}$ Future researchers should focus on qualitative studies exploring cultural factors associated with screening participation. For the factor of perceived barriers, the tool measuring it used by included studies mainly focused on individual burden without a systematic perspective. Identifying barriers to early detection is a vital component of breast cancer control programme. ${ }^{47}$ Future studies should explore a broader understanding of barriers to breast cancer screening with a qualitative design method.

\section{Limitations}

There are several limitations in this review. First, the level of evidence from the included studies was low, with all of the included studies being cross-sectional studies. Second, all studies used self-reported data, which may lead to the over-reporting or under-reporting of screening participation. Future studies should combine self-reporting with medical record reviews to ensure accuracy. Third, there was a lack of consistency in the definitions and measurements of related factors as well as in the screening method and reference period between the screening visit and the questionnaire assessment across the studies. The observed heterogeneity makes the comparison of results across studies difficult and limits our ability to make recommendations regarding strategies to facilitate participation in breast cancer screening. Future studies with prospective methodologies using consistent measurement could provide strong evidence.

\section{CONCLUSIONS}

In summary, by synthesising findings from quantitative studies, this systematic review offered a broad perspective on the factors influencing breast cancer screening participation among women in mainland China. The findings of this review have several implications for health practice and research. To reduce disparities in attending breast cancer screening among women in mainland China, multistrategy interventions tailored to certain geographical regions and health beliefs should be developed. In order to promote breast cancer screening participation among mainland Chinese women, more research with rigorous methodologies is also needed to fully understand the factors associated with breast cancer screening participation. Future studies should make use of data 
obtained from healthcare organisations and the public health surveillance system and apply a longitudinal study design to examine the factors related to breast cancer screening participation. Future studies should focus on multiple levels to understand the biological, psychological, sociocultural and environmental factors influencing breast cancer screening participation based on a theoretical framework.

Contributors ZW, CN and YL designed the study and wrote the protocol. ZW and $\mathrm{CN}$ wrote the search strategy and undertook the literature searches. ZW and CN conducted study screening, data extraction, quality assessment and data analysis. YL resolved disagreements during study screening, data extraction and quality assessment. All authors contributed to the interpretation of the findings. ZW wrote the draft of the manuscript. FL, CN, YL, BS and XL provided critical revisions to the article, and all authors approved the final version of the article to be published.

Funding This work was supported by Research Grant of School of Nursing, China Medical University (grant number 2017HL-14).

Competing interests None declared.

Patient consent for publication Not required.

Provenance and peer review Not commissioned; externally peer reviewed.

Data availability statement All data relevant to the study are included in the article or uploaded as online supplementary information.

Open access This is an open access article distributed in accordance with the Creative Commons Attribution Non Commercial (CC BY-NC 4.0) license, which permits others to distribute, remix, adapt, build upon this work non-commercially, and license their derivative works on different terms, provided the original work is properly cited, appropriate credit is given, any changes made indicated, and the use is non-commercial. See: http://creativecommons.org/licenses/by-nc/4.0/.

\section{REFERENCES}

1. Siegel RL, Miller KD, Jemal A. Cancer statistics, 2018. CA Cancer J Clin 2018;68:7-30.

2. Fan L, Strasser-Weippl K, Li J-J, et al. Breast cancer in China. Lancet Oncol 2014;15:e279-89.

3. Fan L, Zheng Y, Yu K-D, et al. Breast cancer in a transitional society over 18 years: trends and present status in Shanghai, China. Breast Cancer Res Treat 2009;117:409-16.

4. Youlden DR, Cramb SM, Yip CH, et al. Incidence and mortality of female breast cancer in the Asia-Pacific region. Cancer Biol Med 2014;11:101-15.

5. American Cancer Society. Breast Cancer Facts \& Figures 2017-2018. Atlanta: American Cancer Society, 2017. https://www.cancer.org/ content/dam/cancerorg/research/cancer-facts-and-statistics/breastcancer-facts-and-figures/breast-cancer-facts-andfigures-2017-2018. pdf. (accessed 7 July 2018).

6. Nelson HD, Fu R, Cantor A, et al. Effectiveness of breast cancer screening: systematic review and meta-analysis to update the 2009 U.S. Preventive Services Task Force recommendation. Ann Intern Med 2016;164:244-55.

7. United States Preventive Services Task Force. Final recommendation statement: breast cancer. Screening 2016 https://www.uspr eventiveservicestaskforce.org/Announcements/News/ltem/finalrecommendation-statement-screening-for-breast-cancer (accessed 7 July 2018).

8. Committee of breast cancer Society CA-CA. China anti-cancer association guidelines for breast cancer diagnosis and treatment (2017 version). China Oncology 2017;27:695-760.

9. Bao H, Wang L, Wang L, et al. Study on the coverage of cervical and breast cancer screening among women aged 35-69 years and related impact of socioeconomic factors in China , 2013. Chin J Epidemiol 2018;39:208-12.

10. $\mathrm{KM} \mathrm{O}$, Taylor $\mathrm{KL}$, Jacobsen $\mathrm{KH}$. Breast cancer screening among Korean Americans: a systematic review. J Community Health 2017;42:324-32.

11. Lin L, Menon U. Breast and cervical cancer screening practices and interventions among Chinese, Japanese, and Vietnamese Americans Frances. Oncol Nurs Forum 2005;32:995-1003.

12. Alexandraki I, Mooradian AD. Barriers related to mammography use for breast cancer screening among minority women. J Natl Med Assoc 2010;102:206-18.
13. Parsa $\mathrm{P}$, Kandiah $\mathrm{M}$, Abdul Rahman $\mathrm{H}$, et al. Barriers for breast cancer screening among Asian women: a mini literature review. Asian Pac J Cancer Prev 2006;7:509-14.

14. Sarma EA. Barriers to screening mammography. Health Psychol Rev 2015;9:42-62.

15. Ahmadian M, Samah AA. A literature review of factors influencing breast cancer screening in Asian countries. Life Sci J 2012;9:585-94.

16. Kwok C, Sullivan G, Cant R. The role of culture in breast health practices among Chinese-Australian women. Patient Educ Couns 2006;64:268-76.

17. Shamseer L, Moher D, Clarke M, et al. Preferred reporting items for systematic review and meta-analysis protocols (PRISMA-P) 2015: elaboration and explanation. BMJ 2015;349:g7647.10.1136/bmj. g7647

18. Thomas DB, Gao DL, Ray RM, et al. Randomized trial of breast self-examination in Shanghai: final results. J Natl Cancer Inst 2002;94:1445-57.

19. Johannesen M, LoGiudice D. Elder abuse: a systematic review of risk factors in community-dwelling elders. Age Ageing 2013;42:292-8.

20. Huang Y, Zhou K, Li H, et al. Knowledge, attitudes, and behaviour regarding breast cancer screening among women from different socio-economic regions in Southwest China: a cross-sectional study. Asian Pac J Cancer Prev 2011;12:203-9.

21. $\mathrm{Mu} \mathrm{H}, \mathrm{Yu} \mathrm{L}$, Li Y, et al. Study on status and influening factors of breast and cervical cancer screening among urban and rural women in Liaoning Province. Chin J of PHM 2015;31:197-8-201.

22. Sha R, Zhao T, Feng R, et al. Health beliefs and breast cancer screening behavior and willingness of community residents in Hefei. Acta Universitatis Medicinalis Anhui 2017;52:993-7.

23. Tian T, Di J, Yang W, et al. A comparison study on status and influencing factors of knowledge, attitudes, and practice related to breast cancer among women in urban and rural areas in 3 provinces of China. Chin J Health Edu 2018;34:14-8.

24. Wang $B, H e M$, Wang $L$, et al. Breast cancer screening among adult women in China, 2010. Prev Chronic Dis 2013;10:E183.

25. Yan J, Wang D, Gao J. Attending situation and influential factors of breast cancer check programme among women in rural areas. Chinese Health Service Management 2017;5:373-5.

26. You H, Gu H, Zhang N, et al. Why hasn't this woman been screened for breast and cervical cancer? - Evidence from a Chinese population-based study. Public Health 2019;168:83-91.

27. Cheng J. Profile and predictors of breast cancer opportunistic screening utilization among female in Tianjin community. [The Master's Thesis. Tianjin: Tianjin Medical University, 2018.

28. Fang Q, Du Y, Gao Q, et al. Analysis on KAP and its influencing factors of breast cancer screening for women in Hangzhou. Chin Prev Med 2017;18:214-8.

29. Li C, Zheng Y, Pan Y, et al. Study on status and influencing factors of breast cancer screening among perimenopausal outpatients. Maternal and Child Health Care of China 2017;32:4740-2.

30. Wu T-Y, Liu Y-L, Chung S. Improving breast cancer outcomes among women in China: practices, knowledge, and attitudes related to breast cancer screening. Int J Breast Cancer 2012;2012:921607.

31. Zhang X. Analysis of breast cancer screening and associated factors among Suzhou women aged over 40 years. Modern Preventive Medicine 2018;45:2554-7.

32. Gang M, Kim Jl, Oh KO, et al. Factors associated with mammography adherence among married Chinese women in Yanbian, China. Asian Pac J Cancer Prev 2013;14:7207-13.

33. Chen Y. Status of early breast cancer screening and effects of nursing intervention on improving early detection of breast cancer among women in Shanghai. [The Master's Thesis. Shanghai: Fudan University, 2007.

34. Chen L, Fang Q, Guo X. Status of early breast cancer screening and its related factors of 729 females in community. Journal of Nursing 2013;20:71-4.

35. Guo S. Cross-sectional study on knowledge-attitude-practice of breast cancer prevention among community women in Urumqi. [The Master's Thesis. Urumqi: Xinjiang Medical University, 2011.

36. Pan L, Li Q, Tang W, et al. Analysis of the factors influencing breast cancer early detection behaviors on the rural women of Jiading district in Shanghai by use of the health belief model. Shanghai Med Pharma J 2013;34:60-4.

37. Wang T. Breast cancer screening behavior intervention based in Gulou district of Nanjing. [The Master's Thesis]. Nanjing: Southeast University, 2015.

38. Song Q-K, Wang X-L, Zhou X-N, et al. Breast cancer challenges and screening in China: lessons from current registry data and population screening studies. Oncologist 2015;20:773-9.

39. Jones TP, Katapodi MC, Lockhart JS. Factors influencing breast cancer screening and risk assessment among young African 
American women: an integrative review of the literature. $J$ Am Assoc Nurse Pract 2015;27:521-9.10.1002/2327-6924.12223

40. Zhang X, Dupre ME, Qiu L, et al. Urban-Rural differences in the association between access to healthcare and health outcomes among older adults in China. BMC Geriatr 2017;17:151.

41. Wu T-Y, Guthrie BJ, Guthrie B, et al. An integrative review on breast cancer screening practice and correlates among Chinese, Korean, Filipino, and Asian Indian American women. Health Care Women Int 2005;26:225-46.

42. Liu L-Y, Wang Y-J, Wang F, et al. Factors associated with insufficient awareness of breast cancer among women in northern and eastern China: a case-control study. BMJ Open 2018;8:e018523.
43. Dinegde NG, Xuying L. Awareness of breast cancer among female care givers in tertiary cancer Hospital, China. Asian Pac J Cancer Prev 2017;18:1977-83

44. Bao Y, Kwok C, Lee CF. Breast cancer screening behaviors among Chinese women in mainland China. Nurs Health Sci 2018:1-7.

45. Lu M, Moritz S, Lorenzetti D, et al. A systematic review of interventions to increase breast and cervical cancer screening uptake among Asian women. BMC Public Health 2012;12:413.

46. Lee S-Y. Cultural factors associated with breast and cervical cancer screening in Korean American women in the US: an integrative literature review. Asian Nurs Res 2015;9:81-90.

47. El Saghir NS, Adebamowo CA, Anderson BO, et al. Breast cancer management in low resource countries (LRCs): consensus statement from the breast health global initiative. The Breast 2011;20:S3-S11. 